\section{GENETIC VARIATION}

\section{Putting causal variants on the map}

single-
nucleotide
resolution
mapping of
natural genetic
variation

Distinguishing the causal relationship between genotype and phenotype is one of the greatest challenges in the field of genetics. Now, a study published in Cell reports a novel approach to systematically and quantitatively map, at single-nucleotide resolution, causal variants responsible for phenotypic changes in Saccharomyces cerevisiae.

Most heritable traits are influenced by many variants of small effect. Model organisms have been used to map causal variants for decades. However, owing to the low number of meiotic crossovers in these inbred models, most haplotype blocks span many polymorphisms that are strongly correlated. This linkage makes it difficult to distinguish causal mutations from neutral variants. Fine mapping of variants can be attempted by functional profiling of candidates within each quantitative trait locus. However, without cellular and molecular studies it remains extremely difficult to determine which variants are responsible for phenotypic variability.

The identification of causal variants in model organisms requires extensive outbreeding or introduction of individual mutations, both of which are lengthy and costly approaches. She and Jarosz tackled this problem using computational modelling to define the requirements for a mating scheme in yeast that enables single-nucleotide resolution mapping of natural genetic variation. The model takes into account the number of generations of inbreeding, the number of individuals that need to be genotyped and the mean distance between polymorphisms. This approach yielded a statistically optimal crossing scheme for precision mapping. By creating a dense recombination map, the authors were able to statistically differentiate individual causal variants from multiple, highly linked passenger mutations in close proximity.

The authors tested their theoretical model by screening for variants that confer resistance to seven different azoles, the most commonly used class of antifungal drugs. Using their framework, six generations of inbreeding would theoretically be enough to produce a dense recombination map with ten meiotic crossovers between each causal mutation and adjacent passenger mutation. This allowed the authors to first identify a single causal gene, UPC2, and then map which single polymorphisms were responsible for pan-azole resistance. The authors expanded this analysis and mapped 370 causal variants associated with 26 different quantitative traits, identifying different types of mutations, such as missense, synonymous and cis-regulatory mutations, that all contribute to a specific phenotype. Looking at their results from an evolutionary viewpoint, the authors propose that missense and synonymous mutations have different evolutionary roles, with missense mutations acting to modify protein functions and synonymous mutations regulating expression levels.

The systematic approach employed by She and Jarosz highlights how a complex combination of different types of genetic polymorphisms contributes to the richness and variability of complex traits.

Carolina Perdigoto, Associate Editor, Nature Communications

ORIGINAL ARTICLE She, R. \& Jarosz, D. F. Mapping causal variants with single-nucleotide resolution reveals biochemical drivers of phenotypic change. Cell 172, 478-490 (2018) FURTHER READING Eilbeck, K. et al. Settling the score: variant prioritization and Mendelian disease. Nat. Rev. Genet. 18, 599-612 (2017) 